\title{
Resolution of optical isomers of 4-amino-p-chlorobutyric acid lactam by co-crystallization $\dagger$
}

\author{
Mino R. Caira, ${ }^{(1)}$ Luigi R. Nassimbeni, ${ }^{(1)}$ Janet L. Scott, ${ }^{(1) *}$ and Alexander F. Wildervanck
}

While inspecting the proofs of the above article, the authors noticed that they had inadvertently inverted the coordinates of the complex 2 . As this is a chiral structure it has been necessary to correct this. Herewith are the corrected versions of Table 3, and Figs. 3-5.
(1) Department of Chemistry, University of Cape Town, Rondebosch 7700 , South Africa.

* To whom correspondence should be addressed.

$\dagger$ This article appeared in the Journal of Chemical Crystallography, Vol. 26, No. 2, pp. 117-122.
Table 3. Final fractional coordinates for $2\left(\times 10^{4}\right)$

\begin{tabular}{|c|c|c|c|c|}
\hline & $x$ & $y$ & $z$ & $U(\mathrm{eq})$ \\
\hline $\mathrm{Cl}(1)$ & $4474(2)$ & $4507(1)$ & $6923(1)$ & $79(1)$ \\
\hline$C(1 A)$ & $5440(4)$ & $4217(4)$ & $5886(3)$ & $47(1)$ \\
\hline$C(2 A)$ & $6478(4)$ & $5044(5)$ & $5747(3)$ & $51(1)$ \\
\hline$C(3 A)$ & $7211(4)$ & $4792(5)$ & $4910(3)$ & $51(1)$ \\
\hline$C(4 A)$ & $6933(4)$ & $3693(4)$ & $4230(3)$ & $45(1)$ \\
\hline$C(5 A)$ & $5876(4)$ & $2900(4)$ & $4384(3)$ & $48(1)$ \\
\hline$C(6 A)$ & $5122(4)$ & $3154(4)$ & $5214(3)$ & $49(1)$ \\
\hline$C(7 A)$ & $7873(4)$ & $3310(5)$ & $3412(3)$ & $52(1)$ \\
\hline$C(8 A)$ & $8426(4)$ & $4428(5)$ & $2810(3)$ & $46(1)$ \\
\hline$N(1 A)$ & $8725(3)$ & $3835(3)$ & $1810(3)$ & $45(1)$ \\
\hline$C(9 A)$ & $8056(4)$ & $2727(4)$ & $1564(3)$ & $42(1)$ \\
\hline$O(1 \mathrm{~A})$ & $8045(3)$ & $2046(3)$ & $722(2)$ & $55(1)$ \\
\hline$C(10 A)$ & $7243(4)$ & $2434(4)$ & $2468(3)$ & $47(1)$ \\
\hline$O(1 T)$ & $10459(3)$ & $4090(3)$ & $50(2)$ & $48(1)$ \\
\hline $\mathrm{O}(\mathrm{ITH})$ & $9152(3)$ & $2620(3)$ & $-960(2)$ & $59(1)$ \\
\hline$C(I T)$ & $10121(4)$ & $3487(4)$ & $-791(3)$ & $38(1)$ \\
\hline$C(2 T)$ & $10808(3)$ & $3643(4)$ & $-1810(3)$ & $37(1)$ \\
\hline $\mathrm{O}(2 \mathrm{~T})$ & $12015(3)$ & $4342(3)$ & $-1574(2)$ & $42(1)$ \\
\hline$C(3 \mathrm{~T})$ & $9829(4)$ & $4267(4)$ & $-2747(3)$ & $39(1)$ \\
\hline $\mathrm{O}(3 \mathrm{~T})$ & $9547(3)$ & $5554(3)$ & $-2443(2)$ & $51(1)$ \\
\hline$C(4 T)$ & $10432(4)$ & $4222(4)$ & $-3807(3)$ & $40(1)$ \\
\hline$O(4 T)$ & $10672(3)$ & $5212(3)$ & $-4295(2)$ & $51(1)$ \\
\hline $\mathrm{O}(4 \mathrm{TH})$ & $10613(4)$ & $3025(3)$ & $-4131(2)$ & $56(1)$ \\
\hline $\mathrm{Cl}(2)$ & $17454(1)$ & $5922(1)$ & $-10334(1)$ & $67(1)$ \\
\hline$C(1 B)$ & $16104(4)$ & $5674(4)$ & $-9621(3)$ & $47(1)$ \\
\hline$C(2 B)$ & $15825(4)$ & $6579(4)$ & $-8866(3)$ & $50(t)$ \\
\hline$C(3 B)$ & $14767(4)$ & $6348(4)$ & $-8291(3)$ & f9(1) \\
\hline$C(+B)$ & $13985(4)$ & $5216(4)$ & $-8465(3)$ & $40(1)$ \\
\hline$C(5 B)$ & $14298(4)$ & $4329(4)$ & $-9239(3)$ & $48(1)$ \\
\hline$C(6 B)$ & $153+7(4)$ & $+550(5)$ & $-9823(3)$ & $52(1)$ \\
\hline$C(7 B)$ & $12800(4)$ & $4092(4)$ & $-7871(3)$ & $40(1)$ \\
\hline$C(8 B)$ & $12878(4)$ & $5729(4)$ & $-6780(3)$ & $51(1)$ \\
\hline$N(I B)$ & $12077(4)$ & $4910(3)$ & $-6159(3)$ & $47(1)$ \\
\hline$O(I B)$ & $11416(3)$ & $2779(3)$ & $-6054(2)$ & $4 S(1)$ \\
\hline$C(9 B)$ & $11955(3)$ & $3681(4)$ & $-6511(3)$ & $35(1)$ \\
\hline$C(10 B)$ & $12576(4)$ & $3556(4)$ & $-7545(3)$ & $39(1)$ \\
\hline
\end{tabular}




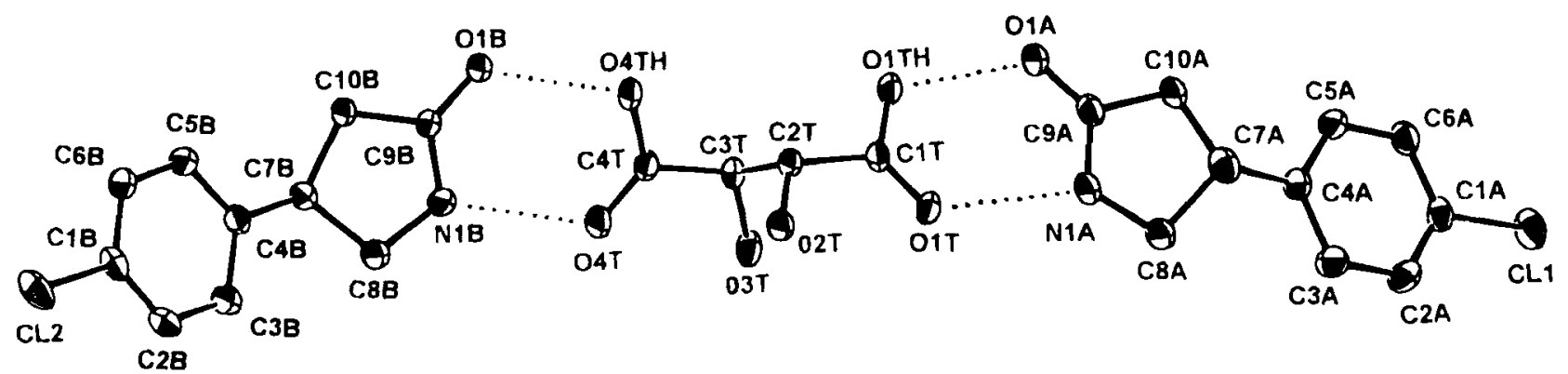

Fig. 3. ORTEP diagram of the complex 2 showing thermal ellipsoids at $30 \%$ probability. Primary hydrogen bonding interactions are indicated as dashed lines.

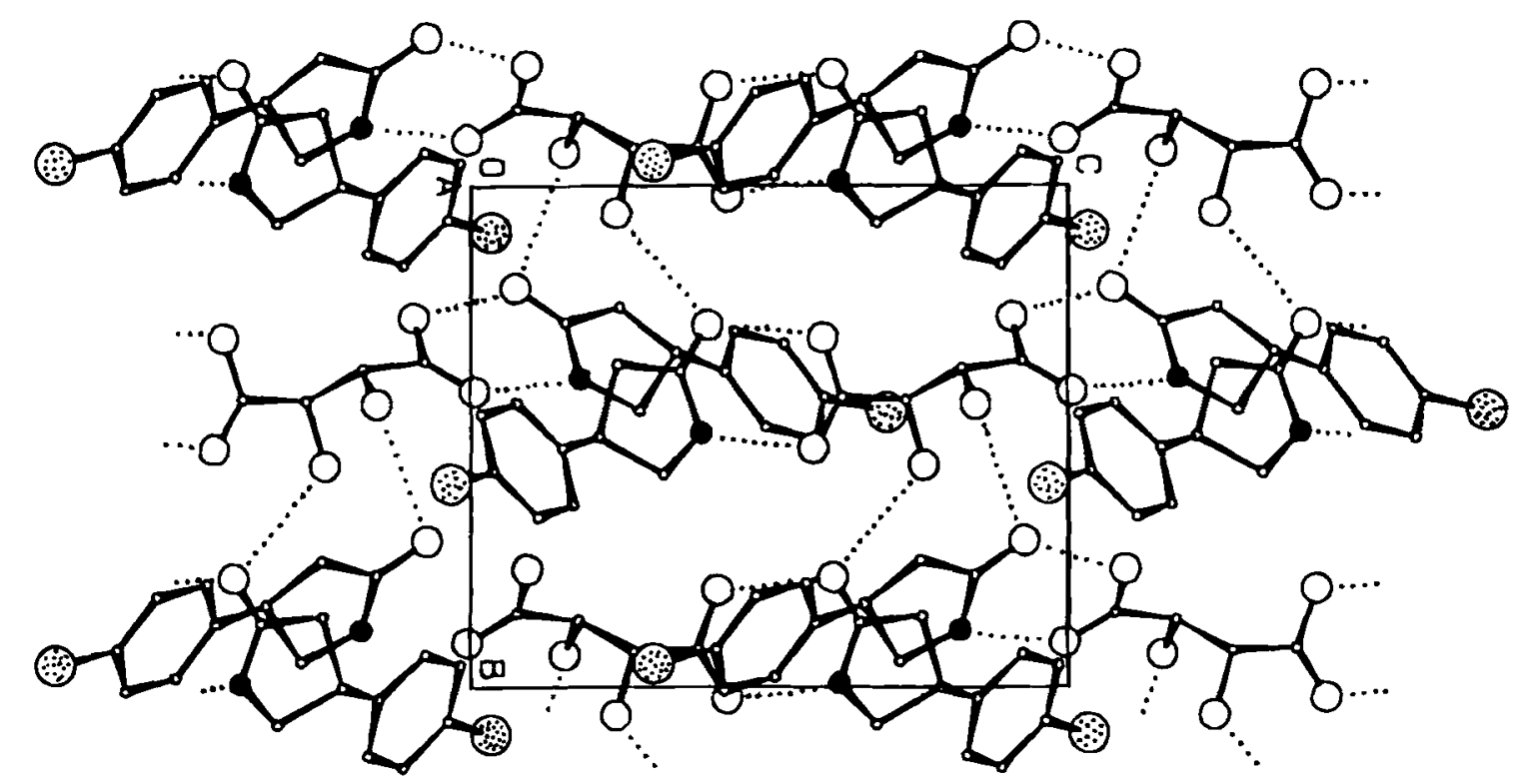

Fig. 4. Packing diagram of the complex 2 viewed as a projection down [100]. Nitrogen atoms are presented as filled circles and chlorine atoms as dotted circles. Hydrogen bonding interactions are indicated by dashed lines.

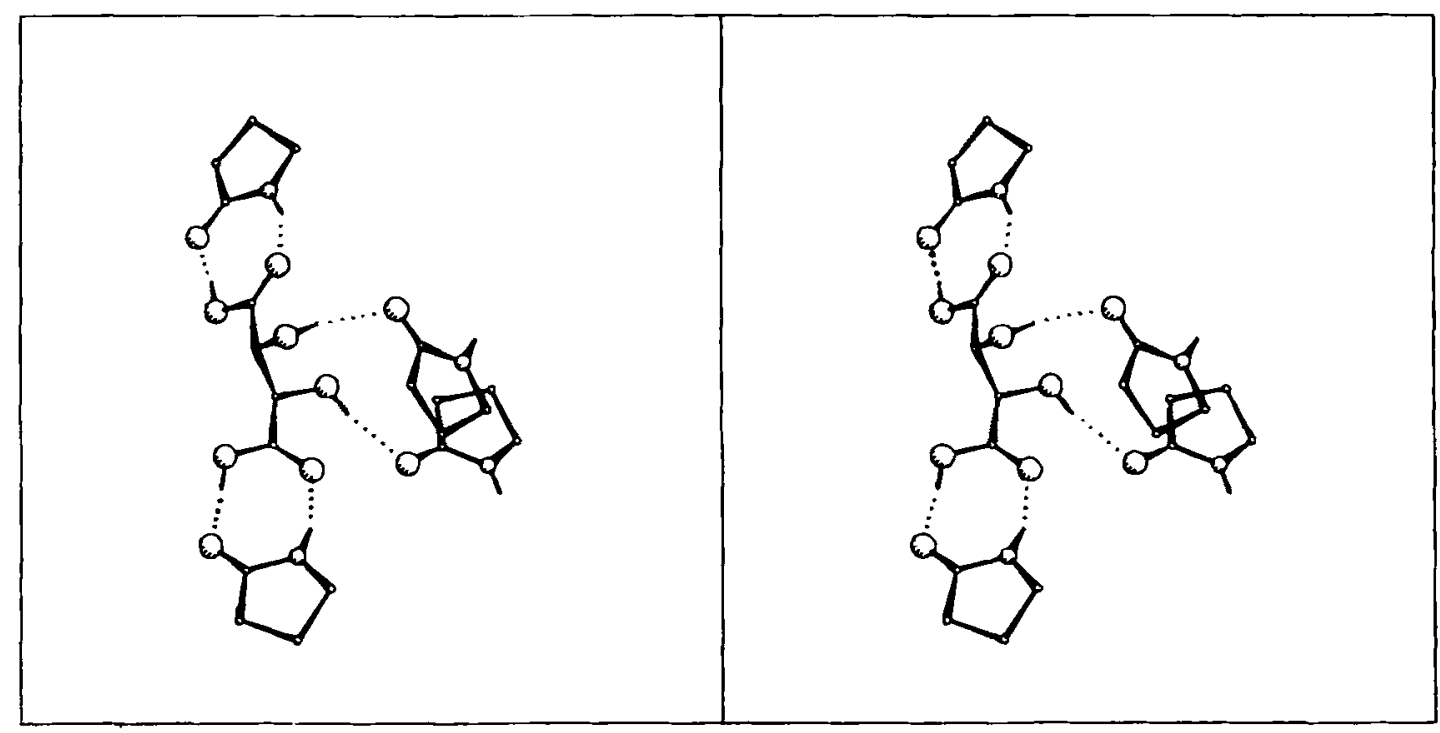

Fig. 5. Stereodiagram showing hydrogen bonding interactions in the 2 complex. Only hydrogen atoms involved in hydrogen bonding interactions are included and the $p$-Cl-phenyl moiety of the lactam molecule is omitted for clarity. 